\title{
LA AFECTIVIDAD EN EL APRENDIZAJE DEL IDIOMA INGLÉS COMO SEGUNDA LENGUA
}

\author{
AFFECTIVITY IN LEARNING ENGLISH \\ AS A SECOND LANGUAGE
}

Martha Romero Zárate ${ }^{1}$

Emma Verónica Santana Valencia ${ }^{2}$

http:/ / orcid.org/0000-0001-8639-3404

Recibido: mayo 12, 2021 - Aceptado: mayo 24, 2021

\section{RESUMEN}

Durante el proceso de aprendizaje de una segunda lengua es posible observar circunstancias diversas que repercuten en él: motivación, interés o desinterés, estrés o cierta sensación de angustia..., que pueden impactar en la adquisición de conocimientos, manifestándose en actitudes positivas o negativas. En el ámbito educativo, la afectividad bien encauzada puede minimizar estas situaciones que impiden al estudiante involucrarse con mayor entusiasmo en su trabajo académico, y en el caso del aprendizaje de una segunda lengua, contribuir a una formación integral.

En este sentido, el presente trabajo presenta una revisión teórica sobre la afectividad en el aprendizaje de una segunda lengua en el contexto universitario y la forma en que este es un soporte para considerar a los estudiantes como seres humanos que requieren de tiempo para integrarse a un ambiente de trabajo, así como de un impulso que los guíe en el proceso de aprendizaje, con paciencia y seguridad, propiciando situaciones de confianza para desarrollar interacciones comunicativas más fluidas con sus pares.

Palabras clave: afectividad, aprendizaje de una lengua, segunda lengua, educación.

1 Doctorante en Educación, Universidad Popular Autónoma del Estado de Puebla (UPAEP), México. Maestría en Educación, Colegio Miguel Hidalgo, Puebla. Docente en la Benemérita Universidad Autónoma de Puebla, México. martha.romero@upaep.edu.mx

2 Doctora en Ciencias para la Familia por el Instituto de Enlaces Educativos de México. Directora Académica de los Posgrados en Investigación y profesora-investigadora, Universidad Popular Autónoma del Estado de Puebla (UPAEP), México. Miembro del Sistema Nacional de Investigadores (SNI) como candidato. Miembro de la Red Internacional de Investigadores y Participantes sobre Integración Educativa (RIIE). Miembro del Consejo Mexicano de Investigación Educativa, AC (COMIE) como candidato. emmaveronica.santana@upaep.mx 


\begin{abstract}
During the process of learning a second language various circumstances can be observed among which motivation, interest or disinterest, stress, anguish that have an impact on the acquisition of knowledge, which are manifested in an attitude which can be positive or negative.

Affectivity in the educational field can minimize these situations that prevent the student from engaging with greater enthusiasm towards their academic work in the case of a second language, it can contribute to an integral formation. In this case, this paperwork presents a theoretical review about affectivity involved in a second language in university students as human beings who require time to feel integrated into a work environment, as well as they need an impulse to be guided with patience and security in order to generate situations of trust to develop more fluent communicative interactions with their peers.
\end{abstract}

Keywords: Affectivity, Learning of a language, Second language, Education.

\title{
INTRODUCCIÓN
}

En el ámbito escolar se han tomado en cuenta diferentes factores que, de alguna manera, impactan en las aulas para generar un conocimiento en los alumnos, que van desde la conducta, el estilo de aprendizaje, el estado de ánimo, la situación económica, la cultural, la edad, las habilidades y las actitudes, etcétera. No obstante, estas últimas no se han considerado del todo. De ahí que valorar su estudio, puede contribuir para conocer la influencia de las actitudes en el aprendizaje de un idioma, especialmente en estudiantes que previamente han contado con un acercamiento formal en el contexto escolar universitario.

La influencia de las actitudes en el contexto escolar, puede manifestarse a través de tres componentes: cognitivo, conductual y afectivo. Se presta especial atención a este último porque involucra las emociones y los sentimientos que de alguna manera impactan en el individuo. Considerar el constructo actitudinal-afectivo, requiere de un análisis profundo para conocer aquellos sucesos que se involucran en el mismo.

El presente documento desarrollará una reflexión teórica sobre la afectividad, como elemento favorecedor del aprendizaje del idioma inglés, en dos sentidos: en un primer momento se citan a los teóricos clásicos (Piaget, Bandura, Ausubel y Vigotsky), los cuales consideran relevante el elemento afectivo para que todo individuo lleve a cabo un aprendizaje. Posteriormente se analizan aquellos teóricos contemporáneos como Gardner, Bisquerra, Pérez, Revee entre otros, los cuales subrayan la importancia de la afectividad, y por último, teóricos como Bonilla, Díaz, Vega, Palacios - por mencionar algunos-, quienes señalan el aporte de la afectividad, involucrada en el aprendizaje de una segunda lengua.

\section{CONSIDERACIÓN DE LA AFECTIVIDAD EN LAS TEORÍAS CLÁSICAS}

La teoría que ha brindado aportes sustanciales en este ámbito -desde la psicología del desarrollo y el enfoque histórico-cultural, hacia cómo el ser humano es capaz de adquirir los conocimientos a través de la interacción sociocultural-, tiene que ver con Lev Vigotsky 
quien, de acuerdo con estudios de Fernando L. González Rey (2000) sobre la afectividad, afirma que esta configura la forma en que el individuo se involucra en un determinado entorno, expresando sentimientos y emociones, parte esencial de su desarrollo socioemocional.

Las vivencias del alumno le apoyarán, pues, en su desempeño cognitivo y afectivo. Aunado a esto, Akhutina (2002) -también sustentado en Vigostsky- encuentra que, desde un punto de vista neuropsicológico, el desarrollo de las funciones afectivas descubre una función inmediata, tanto en los centros subcorticales más antiguos, como en las áreas cerebrales nuevas, además de que en los lóbulos frontales se desarrollan más tardíamente en comparación con todas las demás. La afectividad es una actividad que se presenta a través de los sentidos del ser humano, y se manifiesta en emociones y sentimientos.

En la década de los setenta se reconoció la importancia del factor social en el desarrollo del individuo; se consideró cómo la herencia genética y la interacción con el medio físico, ayudarían al sujeto a construir su conocimiento, resaltándose la afectividad como fuerza motora de la inteligencia y de la conducta (Rodríguez, citado en Vielma y Salas, 2000). Así también, se señalaban a la inteligencia y la afectividad como dos factores relacionados estrechamente en la adquisición de un conocimiento. Vielma y Salas (2000) encontraron que Piaget -conocido como el padre de la epistemología genética, relacionada con la generación de los nuevos conocimientos, a partir del desarrollo de estructuras y mecanismos funcionales en el desarrollo evolutivo humano- reconocía que la inteligencia y la afectividad, estimulan al individuo a generar aprendizajes, de la misma forma en que pueden perturbarlo. Esto en función de una estimulación favorable o desfavorable, pero sin alterar la función cognitiva del mismo, pues la afectividad es un conjunto de emociones y sentimientos. Sin embargo, al relacionarse un proceso cognitivo con un adecuado estímulo afectivo, el individuo generará un mayor progreso positivo en el proceso del aprendizaje (Pérez, 2017). En este sentido, las acciones de un individuo pueden analizarse desde dos dimensiones: afectiva (energética) y cognitiva (asimilación-acomodación) que, al relacionarse, interactúan propiciando aprendizajes más óptimos (Torres y García, 2013).

Una apreciación más hacia la afectividad se observa en Bandura, psicólogo experto en la teoría del aprendizaje social quien, de acuerdo con Vielma y Salas (2000), orienta sus investigaciones sobre el aprendizaje y la socialización a través de modelos -es decir, de patrones o adultos que tienen un papel o una influencia en el aprendizaje del niño o joven-, que permiten la adquisición de conocimientos en el individuo. De acuerdo con sus estudios, dichos modelos no solo son un vehículo importante para la difusión de ideas, valores y estilos de conducta dentro de una sociedad, sino que también poseen una influencia generalizada en los cambios transculturales. Asimismo, reconoce que para llevar a cabo tales modelos, el individuo requiere de la observación, así como de una alta actividad afectiva y cognitiva para lograr dicho proceso.

El aprendizaje de los niños se origina en razón de la observación y el seguimiento de los patrones de padres a hijos, reforzando los conocimientos que adquieren, además de los valores, las conductas y actitudes. Existen cinco funciones del refuerzo vicario: informativa; motivacional; del aprendizaje emocional; de la evaluación, y de la capacidad de ser influido (Ramos Crespo, s/f).

Si se considera que para el aprendizaje del niño o joven es importante recibir información a través de patrones, también es preciso que se sienta motivado, en función de sus emociones y sentimientos -íntimamente ligados con la afectividad-, pues el niño o joven se siente más estimulado a aprender y adquirir nuevos conocimientos, sobre todo cuando percibe cierto reconocimiento en su desempeño. En este sentido, el joven universitario, al encontrarse motivado, tendrá estímulos que lo impulsen a adquirir los conocimientos. 
Ausubel, psicólogo y pedagogo que aborda el aprendizaje significativo, menciona (Viera, 2003) que el individuo genera su aprendizaje sustentado en conocimientos previos que le servirán de soporte para generar uno nuevo, facilitando que ese aprendizaje sea significativo. Además, distingue tres condiciones:

- Aprendizaje representacional: En él se asignan significados a determinados símbolos (palabras) y se identifican los símbolos con sus referentes (objetos, eventos, conceptos).

- Aprendizaje de conceptos: Los conceptos representan regularidades de eventos u objetos y se representan también por símbolos particulares o categorías y, a su vez, representan abstracciones de atributos esenciales de los referentes.

- Aprendizaje proposicional: En este tipo de aprendizaje la tarea no es aprender un significado aislado de los diferentes conceptos que constituyen una proposición, sino los significados de ella como un todo.

Para que un aprendizaje sea significativo, se requiere de un suceso o de un objeto con el cual el individuo haga referencia, convirtiéndose en un símbolo o símbolos para realizar dicha referencia; el aprendizaje no se manifiesta de forma aislada: se presenta como un todo. Ausubel (Viera, 2003) señala que, en este aprendizaje, el sujeto requiere de una disposición positiva en sus procesos motivacionales y afectivos, de modo que agregue a la relación de estas tres condiciones, la generación de una disposición emocional y cognitiva, resultando en un aprendizaje significativo. Por último, no existe aprendizaje sin una estructura cognitiva a la par de una actitud afectiva y motivacional por parte del individuo.

En el siguiente esquema se observa la relación afectividad y cognición para crear aprendizajes significativos, ambos ligados por una interacción que permite adquirir conocimientos nuevos; es decir, se requiere de una estructura cognitiva previa, más un elemento motivador y afectivo que lo impulse a adquirir los conocimientos.

Figura 1. Interacción entre afectividad y estructura cognitiva

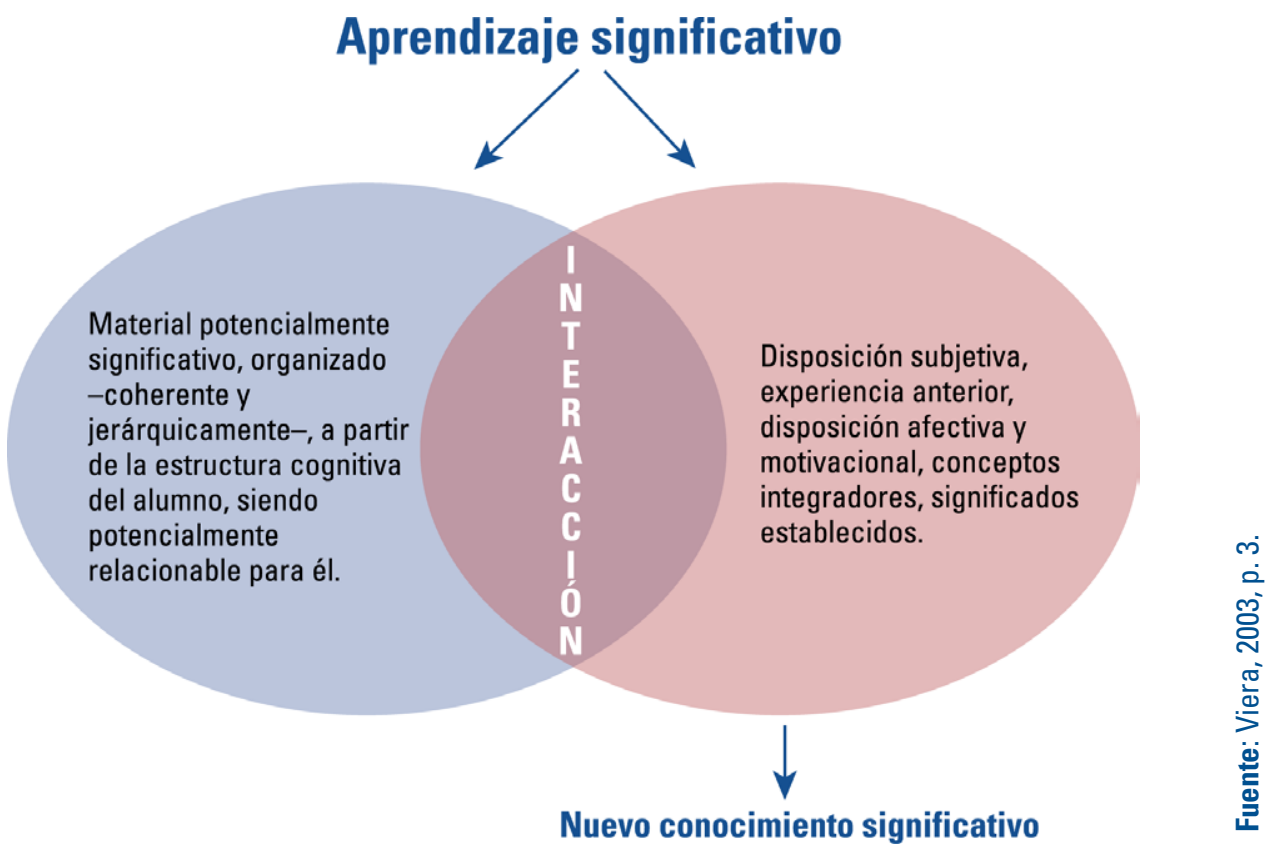


Hasta aquí se ha podido apreciar el aporte de algunos teóricos clásicos quienes, en sus investigaciones, aluden a que la afectividad es relevante para generar nuevos conocimientos; ya sea que hayan sido creados considerando: el contexto del individuo con el medio sociocultural, o a través de ciertos modelos o patrones que le permiten una formación; la afectividad como elemento primordial para motivarlo a adquirir un conocimiento, interviniendo además la actividad afectiva y cognitiva; la importancia de la relación del desarrollo de la inteligencia, los elementos genéticos y el medio social, bajo un ambiente de afecto para el desarrollo y aprendizaje; y el conocimiento previo que, a través de la motivación y el afecto, detonen uno nuevo, convirtiéndolo en significativo.

Aunque las aportaciones de los teóricos antes mencionados, no enfocan sus estudios en el componente afectivo, sí reconocen que bajo condiciones óptimas, el individuo se apropia del conocimiento a través de experiencias y, así, la información recibida y almacenada, se convierte en un aprendizaje que se manifiesta en habilidades, valores y actitudes.

\section{LA AFECTIVIDAD A TRAVÉS DE LAS TEORÍAS CONTEMPORÁNEAS}

Es de gran valor considerar tanto las contribuciones de teóricos clásicos, como de los contemporáneos que también valoran la afectividad como elemento detonador del aprendizaje. De ahí que el aporte en relación con el desarrollo de las inteligencias múltiples es de gran relevancia. En un estudio de Pérez y Beltrán (2006), se mostró que el desenvolvimiento de la inteligencia se fundamenta en dos aspectos, uno biológico y el otro cultural, y que los aprendizajes se concretan con las experiencias. El estudio argumenta que la inteligencia no se desarrolla automáticamente: más bien algo la detona y refuerza con base en el contexto del individuo, y requiere de un fuerte impulso afectivo que lo impacte y lo mueva a llevar a cabo determinada acción.

Gardner, psicólogo conocido por sus investigaciones en el análisis de las capacidades cognitivas y por haber formulado la teoría de las inteligencias múltiples (1998), distingue siete inteligencias múltiples: musical, cinético-corporal, lógico-matemático, lingüística, espacial, interpersonal e intrapersonal. Cada ser humano logra desplegar algunas de estas, que lo hace ser especial frente a los demás. Gardner subraya que las dos últimas deberían considerarse en el ámbito educativo, ya que están vinculadas a las emociones y el afecto; su ausencia resultaría en una atrofia del desarrollo personal y social, originando un analfabetismo emocional que puede derivar en conflictos, violencia, enfrentamientos, suicidios, ansiedad, estrés, depresión, etcétera. Con base en esto, Trianes y García (2002), afirman que para afrontar estos comportamientos, se requiere una educación para la convivencia que incluya programas que contemplen los ámbitos socioemocional y moral, con objetivo de mejorar las relaciones sociales, para que el elemento afectivo contribuya a una educación integral que prevenga conflictos.

Por tanto, el estudio de las inteligencias múltiples toma en cuenta el elemento afectivo, pues es condicionante del aprendizaje -sobre todo cuando se le vincula con la inteligencia interpersonal e intrapersonal-, e influye drásticamente en las emociones del ser humano, de modo que es primordial concederle su esencial protagonismo en el ámbito educativo. Bisquerra (2011), psicólogo y pedagogo, señala que la educación emocional supone pasar de la 
educación afectiva a la educación del afecto, colocando este en el proceso educativo e impartiendo conocimientos teóricos y prácticos sobre él. Una forma de realizarlo sería a través de su aplicación en el currículum para una educación emocional. En este sentido, la inteligencia emocional se concibe como la conexión entre sentimientos y pensamientos, que se encuentran conectados con las regiones emocionales y ejecutivas del cerebro: fisiológicamente, lo que concierne a la enseñanza y aprendizaje. Bisquerra (2012) agrega que la inteligencia emocional es la habilidad del ser humano para tomar conciencia de sus propias emociones, así como de la capacidad para regularlas.

Asimismo, Pérez (2019), argumenta en su investigación que las emociones se originan en razón de las circunstancias de acuerdo con el contexto en el cual se desarrollan, que además impactan en los individuos, y que son estos quienes determinan si las reciben de forma positiva o negativa. Las emociones se encuentran siempre presentes en la vida de los seres humanos y son estos quienes determinan si las asumen de forma favorable o no. El estado emocional de la persona influye en su toma de decisiones; si un estado emocional es manejado adecuadamente por el individuo, de manera que no le afecte del todo, le permitiría tener una visión más clara de sus elecciones.

Revee (citado en Pérez, 2019), psicólogo investigador de la motivación y la emoción, menciona que las emociones comprenden tres funciones principales: adaptativas, sociales y motivacionales, las cuales se describen así:

- Adaptativas: preparan al organismo para afrontar una realidad, desde el contexto donde se ubica el individuo.

- Sociales: la interacción social se manifiesta para mantener relaciones comunicativas favorables, con el fin de mantener saludables lazos socioafectivos.

- Motivacionales: situaciones internas y externas que ejercen una influencia en la persona para determinar su postura ante la vida.

En virtud de ello, si se enfoca la atención de las emociones en la educación, puede comprenderse el por qué, en situaciones de estrés, algunos estudiantes son capaces de afrontar sus emociones, mientras que otros tienen dificultad para hacerlo.

Durand, Daura y Sánchez (2018), en su trabajo de investigación, indican que cuando los aprendices se encuentran en situaciones de estrés, perciben un desequilibrio emocional y es importante que aprendan a afrontar dichas situaciones de forma constructiva para adaptarse a las adversidades, dependiendo de los contextos en los cuales se ubiquen. Y agregan que dicho afrontamiento les sirve de apoyo para que, con base en su experiencia, sobrelleven situaciones de estrés, de forma que aprendan a modificar condiciones para mantener las emociones negativas al margen y que no les afecten; esto les permitirá una mejor adaptación ante escenarios que pudieran perjudicarles.

Considerar el manejo de emociones en las circunstancias adversas, depende de la actitud con la que se enfrenten, y para el caso de los estudiantes universitarios, es vital apoyarles con estrategias capaces de canalizar sus emociones y tomar decisiones acertadas con claridad. En este sentido se aprecia que, para obtener aprendizajes favorables en el ámbito educativo, se requiere pensar en una educación de emociones, para regular sentimientos y pensamientos. Esa regulación contribuirá a la formación del ser humano para enfrentar situaciones existenciales que, de alguna manera, impactan en su contexto. De Souza Barcelar (2011), enfatiza que la realización humana no solo depende del coeficiente intelectual o del grado de estudios 
académicos; el factor más importante es lograr un nivel de inteligencia emocional: conocimiento y regulación de emociones para solucionar situaciones de vida.

Si los docentes las consideran como parte de la formación integral académica de los aprendices, puede formarse en ellos una capacidad de adaptación y resiliencia que los conduzca a tomar decisiones más maduras que, por ende, impacten en una actitud afectiva más armoniosa que contribuya y motive hacia nuevos aprendizajes.

\section{LA AFECTIVIDAD Y EL APRENDIZAJE EN LA SEGUNDA LENGUA}

En el ámbito educativo, el estudio de las inteligencias múltiples significó un punto de inflexión para comprender la manera en cómo los seres humanos asimilan aprendizajes, cómo pueden apropiarse de ellos y cómo llevarlos a la práctica para desarrollarse en la vida. Esto dependerá del tipo de inteligencia que cada uno despliegue, así como también el considerar su estudio en el aprendizaje de alguna lengua. Reflexionar sobre estas inteligencias, ha brindado a los estudiantes de segundas lenguas, la oportunidad de valorar las emociones que intervienen en este proceso: autoestima, inhibición o desinhibición al momento de expresarse, o interactuar con otros aprendices o con el propio profesor, son elementos claves para el éxito en el desarrollo de destrezas comunicativas (Gabarrón, 2019).

Paralelamente a esta concepción, Bonilla y Díaz (2019) afirman que un estudiante motivado puede lograr éxitos en el aprendizaje de una segunda lengua. Para ello deben reunir tres características:

1. Deseo por aprender la lengua.

2. Disposición por esforzarse en medio del proceso o la actividad de aprendizaje.

3. Disposición para sostener la actividad de aprendizaje.

Si un aprendiz no reúne estos elementos, difícilmente un docente podría alcanzar aprendizajes efectivos en dicho proceso y, por lo tanto, lograr los objetivos planeados, pues es un elemento primordial para que el aprendizaje sea óptimo, de manera que el interés que el discente disponga -además de una motivación positiva- generará un aprendizaje favorable, interviniendo distintos elementos afectivos como ya se señaló.

La afectividad en el aprendizaje de una segunda lengua, cobra relevancia al mencionar que esta interviene durante el proceso de asimilación de conocimientos a través de la motivación y la creación de un ambiente favorable, donde el alumno esté cómodo para interactuar con los demás sin sentir estrés, ansiedad ni rechazo por pensar que no puede, o bien que él no es capaz de adquirir los conocimientos para expresarse. En una investigación realizada por Palacios (2020), se demostró que la afectividad en la enseñanza despierta el interés por el aprendizaje de la lengua, además se refuerzan las habilidades cognitivas, desarrollando la capacidad de interacción, y favoreciendo una elevada autoestima y la adecuada toma de decisiones.

En este sentido, Vega (2018) señala que el elemento afectivo debería estar presente en las metodologías actuales para la enseñanza de las lenguas, reconociendo su importancia, pues el aprendiz se desenvolvería mejor al sentir confianza en sí mismo para estructurar mejor 
sus ideas en otro idioma. Pensar que las metodologías existentes añaden afectividad en la enseñanza, podría significar una reflexión en los estilos de aprendizaje futuros, valorando que es un proceso que contribuye a la formación socioemocional del alumno al desarrollarse no solo cognitivamente, sino también emocionalmente.

Debe considerarse el elemento afectivo en la enseñanza de las lenguas, para establecer y valorar estrategias efectivas, como la gramática afectiva: este enfoque, de acuerdo con Méndez Santos (2016), pretende que un aprendizaje bajo un ambiente positivo obtenga estabilidad emocional y mejore la retención del aprendizaje input (información que se recibe) y output (información que se produce). Cabe mencionar que involucrar a los aprendices en situaciones relajadas para la interacción, junto con la práctica de las estructuras gramaticales -donde dichos aprendices se encuentran más concentrados en recordar la función de las mismas-, puede lograr interacciones más fluidas en las situaciones comunicativas. Méndez Santos (2016), argumenta que este enfoque favorece resultados aceptables en el aprendizaje de una segunda lengua, como se describe a continuación:

1. La percepción de la experiencia de aprendizaje de la gramática es más positiva por parte del alumnado.

2. Es mejor la actitud hacia la lengua, la cultura meta y su aprendizaje.

3. La experiencia del aprendizaje se produce en un ambiente emocional estable, relajado y lejos de amenazas que impidan la interiorización de contenidos.

4. La memorización del input es mayor.

5. La sensación de eficacia y de control aumenta.

6. La voluntad del estudiante de producir output es mayor.

Estos alcances se han obtenido gracias a la colaboración de docentes que por años han aplicado este enfoque. De este modo se aprecia su participación por generar cambios significativos en el proceso de enseñanza-aprendizaje.

Valorar el rol del profesor en este proceso, involucra la actitud que él asume ante la enseñanza de una segunda lengua con sus alumnos, al concientizar si se sienten estresados, con ansiedad o con cierta timidez. De ahí la importancia del rol del docente ante estas situaciones que le permitan crear un ambiente favorable, promoviendo un clima emocional dirigido al logro de aprendizajes significativos (Albuja, 2018). Es, pues, esencial considerar el rol del docente como propiciador de situaciones amigables para trabajar emociones que influyen en aprendizajes óptimos, pero también en el currículum educativo, a fin de formar seres humanos íntegros con la capacidad de alcanzar aprendizajes académicos y tomar decisiones acertadas que impacten en su vida, esto gracias a una estabilidad emocional trabajada durante su vida estudiantil.

Rodríguez, García, García y Mata (2020), mencionan que la actitud que el docente asuma hacia su labor académica repercute en cómo los estudiantes la perciben, y cómo esta influye en el proceso del aprendizaje. Si el docente ofrece a sus estudiantes estrategias atractivas para desarrollar las habilidades de comunicación oral y escrita, estas pueden realizarse con confianza y motivación; de esta manera, podrá practicar diálogos y algunas estructuras orales. También agregan que los aprendices se sentirán incentivados a desarrollar las actividades propuestas en el aula con una actitud positiva y mejor desempeño en el aprendizaje del idioma. Cuando ellos perciben una actitud positiva de su maestro, sienten empatía al 
involucrarse en un ambiente que los motiva a realizar sus tareas; pueden experimentar una autoestima que les permita involucrarse en el aprendizaje, y por lo tanto, esto los impulsa a desenvolverse mejor.

De acuerdo con Estupiñan (2020), la involucración del estudiante en las clases de segunda lengua tiene que ver con su propio tipo de motivación y con cómo son motivados en el aula por su profesor -con dinámicas atractivas y creativas-, así como con la forma positiva para integrarse. Es decir, se trata de dos tipos de motivación que influyen en los aprendices: intrínseca y extrínseca. La primera se relaciona con el interés, la voluntad, la confianza del aprendiz; la segunda, influye en las experiencias y condiciones sociales. En este sentido, el docente puede involucrar estímulos afectivos para despertar la atención y el interés de sus alumnos, reforzando estos con el reconocimiento del avance de los mismos e impulsándolos a continuar en el proceso de aprendizaje. Por otro lado, una motivación negativa -alentada por el temor a recibir un estímulo desagradable- puede afectar el desempeño de los estudiantes de modo que les genere ansiedad, falta de interés para participar en las actividades, inseguridad y baja autoestima.

En relación con la afectividad se encontró en una investigación de De Villa (2018), que su papel en la conciencia lingüística ha tenido un impacto de suma relevancia en los estudiantes, pues refuerza emotivamente los caminos neuronales en múltiples áreas del cerebro, logrando un proceso significativo en el lenguaje. Cuando un estudiante se siente motivado a interactuar en las actividades que involucran la práctica del idioma, se vuelve más participativo sin el temor de sentirse juzgado; exterioriza una autoestima acorde para el aprendizaje pues halla, en esta, un impulso para involucrarse en el trabajo colaborativo; lejos de aislarse o cohibirse, muestra una actitud positiva y participativa, expresándose con mayor libertad y realizando sus actividades con más seguridad.

Por otro lado Quintero, Santiago y Veitia, (2021), señalan que el docente debe proveer procesos sanos que generen una dinámica cerebral fluida, los cuales derivarán en un estado de ánimo feliz, ya que estimulan la formación de valores humanos tales como la solidaridad, el amor así mismo y a los demás. Cuando un estudiante es motivado positivamente, su estado emocional permite que su función cerebral esté en armonía con el contexto en el cual se mueve; esto contribuye a que conforme aprendizajes más fluidos y, por lo tanto, su participación en el aula sea más productiva al sentirse en una zona y ambientes propicios, donde la ansiedad o el miedo al ridículo no sean obstáculos para realizar sus tareas y actividades.

En una investigación, España (2020) encontró que los cursos de lenguas provocan reacciones de ansiedad que pueden obstruir la adquisición de la lengua, por lo que sugiere que los docentes sean conscientes del proceso de dicho aprendizaje, no solo involucrándose en aspectos lingüísticos y cognitivos, sino también afectivos. También recomienda que apliquen técnicas para reducir los niveles de ansiedad que experimentan los alumnos. Es importante mencionar que cuando un alumno no es atendido al momento de presentar rasgos de miedo, ansiedad e inseguridad en una clase de inglés, puede sentirse excluido e inseguro para participar abiertamente. La labor del maestro será elegir estrategias no solo acordes con la temática en cuestión, sino también con aquellas que permitan que los alumnos se sientan involucrados en un ambiente de confianza y armonía para interactuar.

Como ya se mencionó, el temor, el estrés, la timidez y la ansiedad en los estudiantes, son factores con que los maestros de la enseñanza de lenguas requieren trabajar, debiendo esforzarse en propiciar situaciones armoniosas y de confianza hacia los aprendices; de lo contrario se estaría creando un ambiente hostil que originará lo que Rabadán y Orgambídez (2018), denominan ansiedad idiomática. Es decir, situaciones donde el alumno obtiene el 
peor rendimiento académico o miedo anticipado por realizar mal alguna actividad oral. En virtud de ello, los autores sugieren que los profesores sean flexibles y respeten los ritmos de aprendizaje, sin autoritarismo ni comparaciones, a fin de facilitar ambientes aceptables para el aprendizaje de la lengua.

Como puede apreciarse, la afectividad involucra aspectos no solo académicos, sino también psicológicos que de alguna manera impactan en el contexto sociocultural del individuo. Si este se encuentra inmerso en ambientes propicios desde su propio contexto, seguramente podrá involucrarse en las labores académicas favorablemente; de lo contrario, si se halla en un ambiente donde se siente inhibido e inseguro, su autoestima se encuentra vulnerable. De ahí la importancia académica al considerar la transcendencia de la afectividad.

\section{CONCLUSIONES}

A través de esta reflexión teórica se aprecia la dimensión de la afectividad en el contexto escolar universitario y cómo puede favorecer al aprendizaje de una segunda lengua. El valorarla favorece el estado de ánimo y el interés del estudiante para tratar situaciones de estrés, como lo menciona Aucancela (2018). Por su parte, el filtro afectivo, propuesto por Krashen (1998), tiene que ver con las causas o circunstancias relacionadas con los estados de ánimo de quienes están aprendiendo: aburrimiento, angustia, miedo, entre otros. Esto puede bloquear el proceso de aprendizaje, lo que significa que en dicho ejercicio se vinculan los procedimientos y las acciones que lo favorecen o entorpecen.

La afectividad, pues, involucra sentimientos y emociones que favorecen o bloquean el aprendizaje en el caso de un idioma. Hoy más que nunca, la afectividad se convierte en un elemento esencial para la convivencia; sin ella, el ser humano no podría relacionarse con los demás, debido a que una convivencia sana y armoniosa debe promoverse no solo en las aulas, sino también en el hogar, la oficina, la empresa...; ello permitirá que las personas que se encuentran en nuestro entorno se sientan tranquilas, relajadas y con una actitud positiva. Estas condiciones son necesarias de tomar en cuenta en el ámbito educativo, de forma particular por los docentes de segundas lenguas, debido a que ellos son los primeros responsables del proceso de enseñanza y aprendizaje.

En el contexto universitario, la afectividad puede considerarse un elemento esencial para motivar al estudiante a estar más en contacto con el aprendizaje del idioma. La afectividad da pauta a que los docentes de la enseñanza de segunda lengua de jóvenes universitarios, se sensibilicen a manejar situaciones y estrategias que apoyen a un acompañamiento más cercano, más humano hacia sus estudiantes, entendiendo que el aprendizaje de un idioma conlleva un proceso y que, en este, la motivación y el afecto cobran un rol importante. El presente artículo abre la posibilidad para generar proyectos y trabajos donde se investigue la enseñanza y el aprendizaje de una segunda lengua en contextos diversos, debido a que la afectividad impacta en situaciones de la vida diaria, así como en el ámbito educativo. 


\section{REFERENCIAS}

Aucancela, P. J. (2018). El filtro afectivo en el desarrollo de la comprensión auditiva del inglés en las/los estudiantes de primero, segundo y tercero de bachillerato del Colegio San Martín de la ciudad de Quito, período lectivo 2017-2018. Tesis de maestría. Facultad de Filosofía, Letras y Ciencias de la Educación, Carrera de Plurilingüe Francés, Universidad Central del Ecuador. http: / / www.dspace.uce.edu.ec/bitstream/25000/17119/1/T-UCE-0010FIL-195.pdf

Albuja, P. (2018). Influencia del estrés en el proceso de aprendizaje del idioma inglés en la academia de guerra aérea. Journal of Education and Human Development, December 2018, vol. 7, no. 4, 56-64. ISSN: 2334-296X (Print), 2334-2978 (Online). http://jehdnet.com/ journals/jehd/Vol 7 No 4 _ December_2018/7.pdf

Akhutina, V. Tatiana. (2002). L.S Vigotsky y A.R. Luria: la formación de la neuropsicología. Revista española de neuropsicología, 4, 2-3, 108-129. https://marxismocritico.files. wordpress.com/2011/10/tatiana_v-_akhutina.pdf

Bisquerra, R. (coord). Punset, Eduard; Mora, Francisco; García Navarro, Esther; López-Cassá, Elia; Pérez-González, Juan Carlos; Lantieri, Linda; Nambiar, Madhavi; Aguilera, Pilar; Segovia, Nieves y Planells, Octavi (2012). ¿Cómo educar las emociones? La inteligencia emocional en la infancia y la adolescencia. Esplugues de Llobregat (Barcelona): Hospital Sant Joan de Déu. https:/ / faros.hsjdbcn.org/adjuntos/2232.1-Faros\%206\%20Cast.pdf

Bisquerra, R. (2011). Diversidad y escuela inclusiva desde la educación emocional. Universidad de Barcelona. https://diversidad.murciaeduca.es/publicaciones/diversa2011/docs/ bisquerra.pdf

Bonilla y Díaz (2019). Incidencia de los factores socio-afectivos en el aprendizaje del inglés. Revista de la Escuela de Ciencias de la Educación, 15(14), vol. 1, enero-junio, 49-64. https://revistacseducacion.unr.edu.ar/index.php/educacion/article/view/405/315

De Villa, A. (2018). El enfoque de la conciencia lingüística: la reflexión crítica en el aprendizaje y enseñanza de segundas lenguas. Revista Didáctica de la lengua / Ensino da lingua. https:// rieoei.org/RIE/article/view/2843/3969

De Souza Barcelar, Lucicleide (2011). Estudio de las emociones: una perspectiva transversal. Contribución a las ciencias sociales. Diciembre 2011. https://www.eumed.net/rev/ cccss/16/lsb.pdf

Durand, J.; Daura, F. y Sánchez, C. (2018). La educación afectiva y emocional, 37-45. https:// www.researchgate.net/profile/DoloresDelRio/ publication/335170119 Estrategias motivacionales y cognitivas Su incidencia en el desempeno academico de alumnos universitarios/links/5d59564e45851545af4c6619/ Estrategias-motivacionales-y-cognitivas-Su-incidencia-en-el-desempeno-academicode-alumnos-universitarios.pdf\#page $=33$ 
España, D. (2020). Ansiedad ante el aprendizaje del inglés como idioma extranjero y percepción de las actitudes del maestro de inglés en estudiantes salvadoreños de educación secundaria. Revista internacional de estudios en educación. https:// riee.um.edu.mx/index. $\mathrm{php} / \mathrm{RIEE} /$ article/view/223/203

Estupiñan, V. (2020). Perspectiva sobre la enseñanza de inglés. Pontificia Universidad Católica del Ecuador, 22. https://repositorio.pucese.edu.ec/bitstream/123456789/2334/1/ ESTUPI\%c3\%91AN\%20MEDINA\%20V\%c3\%8dCTOR\%20HUGO.pdf

González Rey, Fernando L. (2000). El lugar de las emociones en la constitución social de lo psíquico: el aporte de Vigotsky. Educ. Soc., vol. 21, no. 71. Campinas. Julio 2000. https:// www.scielo.br/j/es/a/K74Tm7bWnR5gmNQNSffsQxp/?lang=es

Gabarrón, A. (2019). La enseñanza de las lenguas extranjeras y la teoría de las inteligencias múltiples de Gardner. Reflexiones sobre la importancia de la emoción en el aprendizaje de lenguas extranjeras. Revista de formación del profesorado e investigación educativa. Magister, 31(2), 19-24. https:/ / dialnet.unirioja.es/ servlet/articulo?codigo=7300768

Gardner, H. (1998). Inteligencias múltiples. Barcelona: Paidós. http:/ /infohumanidades.com/ sites / default/files / a puntes / GARDNER\%20inteligencias\% 20multiples\%20las\%20 siete $\% 20$ inteligencias $0 . p d f$

Krashen. D. (1998). The natural approach. Prentice Hall Europe. University of Southern California. http://www.sdkrashen.com/content/books/the natural approach.pdf

Méndez Santos, M. C. (2016). Gramática afectiva en contextos de instrucción formal de español como lengua extranjera ELE. En Méndez Santos, Ma. del Carmen (coord). Nuevas perspectivas en la enseñanza del español como lengua extranjera. Hesperia, XIX(2), 51-84. http:/ / revistas.webs.uvigo.es/index.php/AFH/article/view/702/687

Palacios, Y. (2020). Enseñanza afectiva en el aprendizaje significativo de los estudiantes de Educación de la Universidad Nacional Federico Villarreal, 2019. Tesis de maestría. Universidad Cesar Vallejo. Lima, Perú. http://repositorio.ucv.edu.pe/bitstream/ handle/20.500.12692/41674/Palacios CDCYR.pdf?sequence=1\&isAllowed=y

Pérez, C. (2019). Relación entre emociones y cognición en la enseñanza del inglés en Educación superior. Trabajo de grado, 27-31. http://repository.unipiloto.edu.co/bitstream/ handle / 20.500.12277/ 6524/ TRABAJO\% 20FINAL\% 20JAVIER\% 20PEREZ\% 20 $\% 281 \% 29$.pdf? sequence $=4 \&$ is Allowed $=\mathrm{y}$

Pérez, J. (2017). El desarrollo afectivo según Jean Piaget. Revista Vinculando. http:/ / vinculando. org/wp-content/uploads/kalins-pdf/singles/desarrollo-afectivo-jean-piaget.pdf

Pérez, L. Beltrán, J. (2006). Dos décadas de «inteligencias múltiples»: Implicaciones para la psicología de la educación. Sección monográfica. Universidad Complutense de Madrid. https:// www.redalyc.org/pdf/778/77827304.pdf

Quintero, B.; Santiago, P. y Veitia, M. (2021). La afectividad en el proceso de enseñanzaaprendizaje del idioma inglés en el contexto universitario. http://scielo.sld.cu/scielo. php?pid=S025743142021000100015\&script=sci arttext\&tlng $=$ pt 
Rabadán, M. y Orgambídez, A. (2018). Ansiedad idiomática en español como lengua extranjera y rendimiento académico en la Enseñanza Superior. Revista de estudios e investigación en psicología y educación. ISSN: 1138-1663; e ISSN: 2386-7418 UDC / UMinho, vol. 5, no.1, 29-35. http:/ / rabida.uhu.es/dspace/bitstream/handle/10272/15193/Ansiedad idiomatica.pdf? sequence $=2$

Ramos Crespo, M. G. (s/f). Teorías para educar mejor en valores: Kohlberg, Vygotski, Bandura, Maslow y otros. Facultad de Ciencias de la Educación, Departamento de Ciencias Pedagógicas, Universidad de Carabobo. http:// servicio.bc.uc.edu.ve/educacion/revista/a8n16/8-163.pdf

Rodríguez, N.; García, G.; García, T. Mata, J. (2020). Factores cognitivos y afectivos en la enseñanza y aprendizaje del inglés como lengua extranjera. Edumecentro, vol 12, no. 2, Santa Clara, abril-junio, 2020. Epub 8-abril-2020. http://scielo.sld.cu/scielo. php?pid=S2077-28742020000200230\&script=sci_arttext\&tlng=en

Torres y García, (2013). Interdependencia del desarrollo cognitivo y afectivo. Aproximaciones desde la epistemología genética para la educación inicial. Revista de Pedagogía. Universidad Central de Venezuela. https://www.redalyc.org/pdf/659/65932613002. pdf

Trianes, M.V. y García, A. (2002). Educación socio-afectiva y prevención de conflictos interpersonales en los centros escolares. Revista Interuniversitaria de Formación del Profesorado, no. 44, agosto, 175-189. https:// www.redalyc.org/pdf/274/27404409.pdf

Vega, I. (2018). El componente afectivo en la enseñanza del español a inmigrantes en contextos escolares. Una propuesta para educación primaria. Tesis de maestría. Universidad de León, España. https:/ / buleria.unileon.es/handle/10612/12626

Vielma, E. y Salas, M. L. (2000). Aportes de las teorías de Vygotsky, Piaget, Bandura y Bruner, paralelismo en sus posiciones en relación con el desarrollo. Revista Educere, ISSN 1316-4910. Universidad de los Andes, Venezuela. https://www.redalyc.org/ pdf/356/35630907.pdf

Viera, T. (2003). El aprendizaje verbal significativo de Ausubel. Algunas consideraciones desde el enfoque histórico cultural. Revista Universidades ISSN, 0041-8935. Unión de universidades de América Latina y del Caribe. Organismo internacional. https:// www.redalyc.org/pdf/373/37302605.pdf 